\title{
TWISTOR SPACES WITH HERMITIAN RICCI TENSOR
}

\author{
JOHANN DAVIDOV AND OLEG MUŠKAROV
}

(Communicated by Jonathan M. Rosenberg)

\begin{abstract}
The twistor space $Z$ of an oriented Riemannian 4-manifold $M$ admits a natural 1-parameter family of Riemannian metrics $h_{t}$ compatible with the almost-complex structures $J_{1}$ and $J_{2}$ introduced, respectively, by Atiyah, Hitchin and Singer, and Eells and Salamon. In the present note we describe the (real-analytic) manifolds $M$ for which the Ricci tensor of $\left(Z, h_{t}\right)$ is $J_{n}$-Hermitian, $n=1$ or 2 . This is used to supply examples giving a negative answer to the Blair and Ianus question of whether a compact almost-Kähler manifold with Hermitian Ricci tensor is Kählerian.
\end{abstract}

\section{INTRODUCTION}

Given a compact symplectic manifold $M$, one can consider the integrals $\int_{M} s d V_{g}$ and $\int_{M}\left(s-s^{*}\right) d V_{g}, s\left(\right.$ resp. $\left.s^{*}\right)$ being the scalar (resp. ${ }^{*}$-scalar) curvature, as functionals on the set of metrics associated with the symplectic structure. D. E. Blair and S. Ianus [3] proved that the critical points of these functionals are the associated almost-Kähler metrics for which the Ricci tensor is Hermitian with respect to the corresponding almost-complex structure. Since the Kähler metrics satisfy this condition, Blair and Ianus raised the question of whether a compact almost-Kähler manifold with Hermitian Ricci tensor is Kählerian. A purpose of this note is to show that the twistor space of a compact oriented Riemannian 4-manifold which is Einstein, self-dual, and with negative scalar curvature supplies a negative answer to the question above.

The twistor space $Z$ of an oriented Riemannian 4-manifold admits a natural 1-parameter family of Riemannian metrics $h_{t}$ (cf., e.g. $[8,9,13]$ ) compatible with the almost-complex structures $J_{1}$ and $J_{2}$ on $Z$ introduced, respectively, by Aityah, Hitchin and Singer [1], and Eells and Salamon [7]. Motivated by the Blair and Ianus result, we consider the problem when the Ricci tensor of $\left(Z, h_{t}\right)$ is $J_{n}$-Hermitian, $n=1$ or 2 , and prove the following theorem:

Theorem. Let $M$ be a connected oriented real-analytic Riemannian 4-manifold. If the Ricci tensor of the twistor space $\left(Z, h_{t}\right)$ is $J_{n}$-Hermitian, then either

Received by the editors August 7, 1989.

1980 Mathematics Subject Classification (1985 Revision). Primary 53C25, 53C15.

(C) 1990 American Mathematical Society $0002-9939 / 90 \$ 1.00+\$ .25$ per page 
(i) $M$ is Einstein and self-dual

or

(ii) $M$ is self-dual with constant scalar curvature $s=12 / t$ and, for each point of $M$, at least three eigenvalues of its Ricci operator coincide.

Conversely, if $M$ is a (smooth) Riemannian 4-manifold satisfying (i) or (ii), then the Ricci tensor of $\left(Z, h_{t}\right)$ is $J_{n}$-Hermitian.

The proof is based on an explicit formula for the Ricci tensor of $\left(Z, h_{t}\right)$ in terms of the curvature of $M$ [4].

Remarks. (1) Let $M$ be an oriented Riemannian 4-manifold. If $M$ is Einstein, self-dual, and with negative scalar curvature $s$, then $\left(h_{t}, J_{2}\right)$ for $t=-12 / s$ is an almost-Kähler structure on the twistor space $Z$ [12]. This structure is not Kählerian since the almost-complex structure $J_{2}$ is never integrable [7]. By the theorem, the Ricci tensor of $\left(Z, h_{t}\right)$ is $J_{2}$-Hermitian, so, if $M$ is compact, $\left(Z, h_{t}, J_{2}\right)$ gives a negative answer to the Blair and Ianus question. Note that the only known examples of such manifolds $M$ are compact quotients of the unit ball in $\mathbf{C}^{2}$ with the metric of constant negative curvature or the Bergman metric (cf., e.g. [13]).

On the other hand the classification of compact connected self-dual Einstein 4-manifolds $M$ with nonnegative scalar curvature $s$ is well known: If $s>$ $0, M$ is the unit sphere $S^{4}$ or the complex projective space $\mathbf{C} \mathbf{P}^{2}$ with their standard metrics $[9,11]$. If $s=0$, the universal covering of $M$ is a $K 3$-surface with a Ricci flat Kähler metric or $M$ is flat [10].

(2) Let $S^{1}$ and $S^{3}$ be the unit spheres of dimensions one and three. Then $M=S^{1} \times S^{3}$ with the product-metric is a non-Einstein manifold satisfying the conditions (ii) of the theorem. Other examples of such manifolds $M$ can be obtained as warped-products of $S^{1}$ and $S^{3}$ (cf. [5]).

\section{Preliminaries}

Let $M$ be an oriented Riemannian 4-manifold with metric $g$. Then $g$ induces a metric on the bundle $\wedge^{2} T M$ of 2-vectors by $g\left(A_{1} \wedge A_{2}, A_{3} \wedge A_{4}\right)=$ $1 / 2 \cdot \operatorname{det}\left(g\left(A_{i}, A_{j}\right)\right)$. Let $\nabla$ be the Riemannian connection of $(M, g)$. For the curvature tensor $R$ of $\nabla$, we adopt the following definition $R(A, B)=\nabla_{[A, B]}-$ $\left[\nabla_{A}, \nabla_{B}\right]$. The curvature operator $\mathscr{R}$ is the self-adjoint endomorphism of $\wedge^{2} T M$ defined by $g(\mathscr{R}(A \wedge B), C \wedge D)=g(R(A, B) C, D)$. The Hodge star operator defines an endomorphism $*$ of $\Lambda^{2} T M$ with $*^{2}=I d$. Hence $\Lambda^{2} T M=\Lambda_{+}^{2} T M \oplus \Lambda_{-}^{2} T M$ where $\Lambda_{ \pm}^{2} T M$ are the subbundles of $\Lambda^{2} T M$ corresponding to the $( \pm 1)$-eigenvalues of $*$. Let $\left(E_{1}, E_{2}, E_{3}, E_{4}\right)$ be a local oriented orthonormal frame of $T M$. Set

$$
\begin{array}{ll}
s_{1}=E_{1} \wedge E_{2}-E_{3} \wedge E_{4}, & \bar{s}_{1}=E_{1} \wedge E_{2}+E_{3} \wedge E_{4}, \\
s_{2}=E_{1} \wedge E_{3}-E_{4} \wedge E_{2}, & \bar{s}_{2}=E_{1} \wedge E_{3}+E_{4} \wedge E_{2}, \\
s_{3}=E_{1} \wedge E_{4}-E_{2} \wedge E_{3}, & \bar{s}_{3}=E_{1} \wedge E_{4}+E_{2} \wedge E_{3} .
\end{array}
$$


Then $\left(s_{1}, s_{2}, s_{3}\right)$ (resp., $\left.\left(\bar{s}_{1}, \bar{s}_{2}, \bar{s}_{3}\right)\right)$ is a local oriented orthonormal frame of $\bigwedge_{-}^{2} T M$ (resp., $\bigwedge_{+}^{2} T M$ ). The block-decomposition of $\mathscr{R}$ with respect to the above splitting of $\wedge^{2} T M$ is

$$
\mathscr{R}=\left[\begin{array}{cc}
s / 6 \cdot I d+\mathscr{W}_{+} & \mathscr{B} \\
t_{B} & s / 6 \cdot I d+\mathscr{W}_{-}
\end{array}\right],
$$

where $s$ is the scalar curvature of $M ; s / 6 \cdot I d+\mathscr{B}$ and $\mathscr{W}=\mathscr{W}_{+}+\mathscr{W}_{-}$ represent the Ricci tensor and the Weyl conformal tensor, respectively. The manifold $M$ is said to be self-dual (anti-self-dual) if $\mathscr{W}_{-}=0 \quad\left(\mathscr{W}_{+}=0\right)$. It is Einstein exactly when $\mathscr{B}=0$.

The twistor space of $M$ is the 2-sphere bundle $\pi: Z \rightarrow M$ consisting of the unit vectors of $\bigwedge_{-}^{2} T M$. The Riemannian connection of $M$ gives rise to a splitting $T Z=\mathscr{H} \oplus \mathscr{V}$ of the tangent bundle of $Z$ into horizontal and vertical components. We further consider the vertical space $\mathscr{V}_{\sigma}$ at $\sigma \in Z$ as the orthogonal complement of $\sigma$ in $\Lambda_{-}^{2} T_{p} M, p=\pi(\sigma)$.

Each point $\sigma \in Z$ defines a complex structure $K_{\sigma}$ on $T_{p} M, p=\pi(\sigma)$, by

$$
g\left(K_{\sigma} A, B\right)=2 g(\sigma, A \wedge B), \quad A, B \in T_{p} M .
$$

This structure is compatible with the metric $g$ and the opposite orientation of $M$ at $p$.

Denote by $\times$ the usual vector product in the oriented three-dimensional vector space $\Lambda_{-}^{2} T_{p} M$. Following [1] and [7], define two almost-complex structures $J_{1}$ and $J_{2}$ on $Z$ by

$$
\begin{array}{cc}
J_{n} V=(-1)^{n} \sigma \times V & \text { for } V \in \mathscr{V}_{\sigma}, \\
\pi_{*}\left(J_{n} X\right)=K_{\sigma}\left(\pi_{*} X\right) & \text { for } X \in \mathscr{H}_{\sigma} .
\end{array}
$$

It is well known [1] that $J_{1}$ is integrable (i.e. comes from a complex constructure on $Z$ ) iff $M$ is self-dual. Unlike $J_{1}$, the almost-complex structure $J_{2}$ is never integrable [7].

As in [9], define a pseudo-Riemannian metric $h_{t}$ on $Z$ by $h_{t}=\pi^{*} g+t g^{v}$, where $t \neq 0$ and $g^{v}$ is the restriction of the metric of $\bigwedge^{2} T M$ on the vertical distribution $\mathscr{V}$. Then $h_{t}$ is compatible with the almost-complex structures $J_{1}$ and $J_{2}$.

\section{Proof of THE THEOREM}

Lemma. The Ricci tensor $c_{Z}$ of $\left(Z, h_{t}\right)$ is Hermitian with respect to $J_{n}$ iff for each point $\sigma \in Z$ one has:

$$
\left(12-t s(p)+6 \operatorname{tg}\left(\mathscr{W}_{-}(\sigma), \sigma\right)\right) \mathscr{B}(\sigma)=0,
$$
where $p=\pi(\sigma)$ and $s$ is the scalar curvature of $M$.

$$
\begin{gathered}
\|\mathscr{R}(\cdot)\|=\text { const on the fibre } Z_{p} \text { through } \sigma . \\
g((\delta \mathscr{R})(X), \sigma \times V)=(-1)^{n+1} g\left((\delta \mathscr{R})\left(K_{\sigma} X\right), V\right)
\end{gathered}
$$


for every $X \in T_{p} M$ and $V \in \mathscr{V}_{\sigma}$. Here $\delta \mathscr{R}$ is the codifferential of $\mathscr{R}$ and $K_{\sigma}$ is the complex structure on $T_{p} M$ determined by $\sigma$ via (2.2).

Proof. If $E \in T Z, X=\pi_{*} E$ and $V$ is the vertical component of $E$, then [4]

$$
\begin{aligned}
c_{Z}(E, E)= & c(X, X)+\operatorname{tg}((\delta \mathscr{R})(X), \sigma \times V)+\left(t^{2} / 4\right)\|\mathscr{R}(\sigma \times V)\|^{2} \\
& -(t / 2)\left\|i_{X} \circ \mathscr{R}_{-}\right\|^{2}+(t / 2)\left\|\left(i_{X} \circ \mathscr{R}\right)(\sigma)\right\|^{2}+\|V\|^{2},
\end{aligned}
$$

where $c$ is the Ricci tensor of $M, i_{X}: \wedge^{2} T M \rightarrow T M$ is the interior product and $\mathscr{R}_{-}$is the restriction of $\mathscr{R}$ on $\bigwedge_{-}^{2} T M$.

We first show that $c_{Z}$ is $J_{n}$-Hermitian on horizontal vectors iff (3.1) holds for every $\sigma \in Z$. In fact, it follows from (3.4) that $c_{Z}$ is $J_{n}$-Hermitian on the horizontal space $\mathscr{H}_{\sigma}$ iff

$$
\begin{aligned}
& 2 c(X, X)-t\|R(\tau) X\|^{2}-t\|R(\sigma \times \tau) X\|^{2} \\
& \quad=2 c\left(K_{\sigma} X, K_{\sigma} X\right)-t\left\|R(\tau) K_{\sigma} X\right\|^{2}-t\left\|R(\sigma \times \tau) K_{\sigma} X\right\|^{2}
\end{aligned}
$$

for $X \in T_{p} M$ and $\tau \in Z_{p}, \tau \perp \sigma$. Fix $\tau \in Z_{p}, \tau \perp \sigma$ and $E \in T_{p} M,\|E\|=1$. Since $K_{\sigma} \circ K_{\tau}=-K_{\sigma \times \tau},\left(E_{1}, E_{2}, E_{3}, E_{4}\right)=\left(E, K_{\sigma} E, K_{\tau} E, K_{\sigma \times \tau} E\right)$ is an oriented orthonormal basis of $T_{p} M$ such that $\sigma=s_{1}, \tau=s_{2}$, and $\sigma \times \tau=s_{3}$, where $s_{1}, s_{2}, s_{3}$ are defined by (2.1). For $X \in T_{p} M$, denote

$$
V_{i}=X \wedge E_{i}-K_{\sigma} X \wedge K_{\sigma} E_{i}, \quad \bar{V}_{i}=X \wedge E_{i}+K_{\sigma} X \wedge K_{\sigma} E_{i}, \quad i=1, \ldots, 4 .
$$

Then

$$
\begin{gathered}
c(X, X)-c\left(K_{\sigma} X, K_{\sigma} X\right)=\sum_{i=1}^{4} g\left(\mathscr{R}\left(V_{i}\right), \bar{V}_{i}\right) \\
\|R(\tau) X\|^{2}-\left\|R(\tau) K_{\sigma} X\right\|^{2}=\sum_{i=1}^{4} g\left(\mathscr{R}(\tau), V_{i}\right) g\left(\mathscr{R}(\tau), \bar{V}_{i}\right) .
\end{gathered}
$$

If $X=\sum_{i=1}^{4} \lambda_{i} E_{i}$, then

$$
\begin{array}{ll}
V_{1}=-\lambda_{3} s_{2}-\lambda_{4} s_{3}, & \bar{V}_{1}=-\lambda_{2}\left(s_{1}+\bar{s}_{1}\right)-\lambda_{3} \bar{s}_{2}-\lambda_{4} \bar{s}_{3}, \\
V_{2}=\lambda_{3} s_{3}-\lambda_{4} s_{2}, & \bar{V}_{2}=\lambda_{1}\left(s_{1}+\bar{s}_{1}\right)-\lambda_{3} \bar{s}_{3}+\lambda_{4} s_{2}, \\
V_{3}=\lambda_{1} s_{2}-\lambda_{2} s_{3}, & \bar{V}_{3}=-\lambda_{4}\left(\bar{s}_{1}-s_{1}\right)+\lambda_{1} \bar{s}_{2}+\lambda_{2} \bar{s}_{3}, \\
V_{4}=\lambda_{1} s_{3}+\lambda_{2} s_{2}, & \bar{V}_{4}=\lambda_{3}\left(\bar{s}_{1}-s_{1}\right)-\lambda_{2} \bar{s}_{2}+\lambda_{1} \bar{s}_{3} .
\end{array}
$$

Substituting (3.6) and (3.7) into (3.5) and then varying $\left(\lambda_{1}, \ldots, \lambda_{4}\right)$, one sees that the identity (3.5) holds iff

$$
(2-\operatorname{tg}(\mathscr{R}(\sigma), \sigma) \mathscr{B}(\sigma))-\operatorname{tg}(\mathscr{R}(\sigma), \tau) \mathscr{B}(\tau)=0
$$

for all $\tau \in Z_{p}, \tau \perp \sigma$. Taking a point $\tau \in Z_{p}$ such that $\tau \perp \sigma$ and $g(\mathscr{R}(\sigma), \tau)=$ 0 , one obtains (3.1). Conversely, assume that the identity (3.1) holds for every $\sigma \in Z$. Fix a point $p \in M$. Then either $\mathscr{B}_{p}=0$ or $12-t s(p)+$ $6 \operatorname{tg}\left(\mathscr{W}_{-}(\sigma), \sigma\right)=0$ for all $\sigma \in Z_{p}$. In the second case, $12-t s(p)=0$ 
since trace $\mathscr{W}_{-}=0$ and therefore $\left(\mathscr{W}_{-}\right)_{p}=0$. So $g(\mathscr{R}(\sigma), \tau)=0$ for every $\sigma, \tau \in Z_{p}, \sigma \perp \tau$. In both cases (3.8) is fulfilled and $c_{Z}$ is $J_{n}$-Hermitian on horizontal vectors.

It is obvious from (3.4) that $c_{Z}$ is $J_{n}$-Hermitian on vertical vectors iff $\|\mathscr{R}(\sigma)\|=\|\mathscr{R}(\tau)\|$ for every $\sigma, \tau \in Z$ with $\pi(\sigma)=\pi(\tau)$ and $\sigma \perp \tau$, which is equivalent to (3.2). Formula (3.4) also shows that $c_{Z}\left(J_{n} E, J_{n} V\right)=c_{Z}(E, V)$ for all $E \in \mathscr{H}_{\sigma}, V \in \mathscr{V}_{\sigma}$ iff (3.3) holds. Thus the lemma is proved.

To prove the theorem, first assume that the Ricci tensor $c_{Z}$ of $\left(Z, h_{t}\right)$ is $J_{n^{-}}$ Hermitian. Then the identity (3.1) of the lemma and the principle of analytic continuation imply that either $\mathscr{B} \equiv 0$ or

$$
12-t(s \circ \pi)(\sigma)+6 \operatorname{tg}\left(\mathscr{W}_{-}(\sigma), \sigma\right) \equiv 0 \quad \text { on } Z .
$$

We shall show that in the first case $M$ is self-dual. Consider $\mathscr{W}_{-}$as a selfadjoint endomorphism of $\Lambda_{-}^{2} T_{p} M, p \in M$, and denote by $\mu_{1}, \mu_{2}, \mu_{3}$ its eigenvalues. Since $\mathscr{B}=0, \mathscr{R}(\sigma)=(s / 6) \sigma+\mathscr{W}_{-}(\sigma)$ for $\sigma \in \Lambda_{-}^{2} T_{p} M$, and the condition (3.2) of the lemma gives $\left|\mu_{1}+s / 6\right|=\left|\mu_{2}+s / 6\right|=\left|\mu_{3}+s / 6\right|$. Moreover, $\mu_{1}+\mu_{2}+\mu_{3}=$ trace $\mathscr{W}_{-}=0$. Hence either $\mu_{1}=\mu_{2}=\mu_{3}=0$ or $\left\{\mu_{1}, \mu_{2}, \mu_{3}\right\}=\{s / 3, s / 3,-2 s / 3\}$. It follows that either $\left\|\mathscr{W}_{-}\right\| \equiv 0$ or $\left\|\mathscr{W}_{-}\right\| \equiv 2 s^{2} / 3$. So we have to consider only the case when $\left\|\mathscr{W}_{-}\right\| \equiv 2 s^{2} / 3$. Since $M$ is Einstein, $\delta \mathscr{W}_{-}=0$ (cf., e.g. [2, $\left.\S 16.5\right]$ ) and Proposition 5, (iii) of [6] gives $\nabla \mathscr{W}_{-}=0$. For every oriented Riemannian 4-manifold with $\delta \mathscr{W}_{-}=0$, one has $[2, \S 16.73]$

$$
\Delta\left\|\mathscr{W}_{-}\right\|^{2}=-s\left\|\mathscr{W}_{-}\right\|^{2}+18 \operatorname{det} \mathscr{W}_{-}-2\left\|\nabla \mathscr{W}_{-}\right\|^{2},
$$

which implies in our case $s=0$. Hence $\mathscr{W}_{-}=0$.

Now assume that the identity (3.9) is satisfied. Then $s=12 / t$ since trace $\mathscr{W}_{-}$ $=0$. Therefore $g\left(\mathscr{W}_{-}(\sigma), \sigma\right) \equiv 0$ which shows that $\mathscr{W}_{-}=0$. Thus $\mathscr{R}(\sigma)=$ $(2 / t) \sigma+\mathscr{B}(\sigma)$ for $\sigma \in Z$, and (3.2) of the lemma is equivalent to $\|\mathscr{B}(\cdot)\|$ being constant on the fibre through each point $\sigma \in Z$. Let $C: T_{p} M \rightarrow T_{p} M, p \in M$, be the Ricci operator and $\left(E_{1}, E_{2}, E_{3}, E_{4}\right)$ an oriented orthonormal basis of $T_{p} M$ consisting of eigenvectors of $C$. Denote by $\lambda_{i}, i=1, \ldots, 4$, the corresponding eigenvalues. Let $\left(\bar{s}_{i}, s_{i}\right)$ be the basis of $\Lambda^{2} T_{p} M$ defined by (2.1). Since $\lambda_{1}+\lambda_{2}+\lambda_{3}+\lambda_{4}=s$ and $\mathscr{B}(X \wedge Y)=C(X) \wedge Y+X \wedge C(Y)-(s / 2) X \wedge Y$, one has $\mathscr{B}\left(s_{1}\right)=\left(\lambda_{1}+\lambda_{2}-s / 2\right) \bar{s}_{1}, \mathscr{B}\left(s_{2}\right)=\left(\lambda_{1}+\lambda_{3}-s / 2\right) \bar{s}_{2}, \mathscr{B}\left(s_{3}\right)=$ $\left(\lambda_{1}+\lambda_{4}-s / 2\right) \bar{s}_{3}$. Therefore $\|\mathscr{B}(\cdot)\|=$ const on $Z_{p}$ iff $\left|\lambda_{1}+\lambda_{2}-s / 2\right|=$ $\left|\lambda_{1}+\lambda_{3}-s / 2\right|=\left|\lambda_{1}+\lambda_{4}-s / 2\right|$, i.e. iff at least three eigenvalues of $C$ coincide.

Conversely, let $M$ be a (smooth) Einstein self-dual 4-manifold. Then $\mathscr{R}(\sigma)$ $=(s / 6) \sigma, \sigma \in Z, \delta \mathscr{R}=0$ (cf., e.g. [2, §16.3]), and the three conditions of the lemma obviously hold. Now, assume that $M$ satisfies the condition (ii) of the theorem. Then (3.1) is obvious and (3.2) follows from the arguments above. Since $s=12 / t$ and $\mathscr{W}_{-}=0$, one has $\delta \mathscr{R}=2 \delta \mathscr{W}=2 \delta \mathscr{W}_{+}([2, \S 16.5])$, so $(\delta \mathscr{R})(X) \in \bigwedge_{+}^{2} T_{p} M$. Hence, (3.3) holds and the theorem is proved. 


\section{REFERENCES}

1. M. F. Atiyah, N. J. Hitchin and I. M. Singer, Self-duality in four-dimensional Riemannian geometry, Proc. Roy. Soc. London Ser. A 362 (1978), 425-461.

2. A. L. Besse, Einstein manifolds, Springer-Verlag, Berlin, 1978.

3. D. E. Blair and S. Ianus, Critical associated metrics on symplectic manifolds, in Nonlinear Problems in Geometry, Contemp. Math. 51 (1986), 23-29.

4. J. Davidov and O. Muškarov, On the Riemannian curvature of a twistor space, preprint series of the ICTP, Trieste, 1988.

5. A. Derdzinski, Classification of certain compact Riemannian manifolds with harmonic curvature and non-parallel Ricci tensor, Math. Z. 172 (1980), 273-280.

6. __ Self-dual Kähler manifolds and Einstein manifolds of dimension four, Compositio Math. 49 (1983), 405-433.

7. J. Eells and S. Salamon, Twistorial construction of harmonic maps of surfaces into four-manifolds, Ann. Scuola Norm. Sup. Pisa Cl. Sci. 12 (1985), 589-640.

8. Th. Friedrich and R. Grunewald, On Einstein metrics on the twistor space of a four-dimensional Riemannian manifold, Math. Nachr. 123 (1985), 55-60.

9. Th. Friedrich and H. Kurke, Compact four-dimensional self-dual Einstein manifolds with positive scalar curvature, Math. Nachr. 106 (1982), 271-299.

10. N. J. Hitchin, Compact four-dimensional Einstein manifolds, J. Differential Geom. 9 (1974), 435-441.

11. __ Kählerian twistor spaces, Proc. London Math. Soc. 43 (1981), 133-150.

12. O. Muškarov, Structures presque hermitiennes sur des espaces twistoriels et leur types, C. R. Acad. Sci. Paris Sér. I Math. 305 (1987), 307-309.

13. A. Vitter, Self-dual Einstein metrics, in Nonlinear Problems in Geometry, Comtemp. Math. 51 (1986), 113-120.

Institute of Mathematics, Bulgarian Academy of Sciences, ul. "Acad. G. Bontchev", BL.8, 1090-Sofia, BuLgaRIA 